\title{
Native oxide formation on pentagonal copper nanowires: A TEM study
}

\author{
Rashad Hajimammadov, Melinda Mohl, Krisztian Kordas* \\ Microelectronics Research Unit, Faculty of Information Technology and Electrical Engineering, University of Oulu, P.O. Box 4500, 90570 Oulu, Finland
}

\section{A R T ICLE INFO}

\section{Keywords:}

Copper nanowires

Penta-twinned crystal

Room temperature oxidation

Growth kinetics

Copper oxides

\begin{abstract}
A B S T R A C T
Hydrothermally synthesized copper nanowires were allowed to oxidize in air at room temperature and $30 \%$ constant humidity for the period of 22 days. The growth of native oxide layer was followed up by high-resolution transmission electron microscopy and diffraction to reveal and understand the kinetics of the oxidation process. Copper oxides appear in the form of differently oriented crystalline phases around the metallic core as a shell-like layer $\left(\mathrm{Cu}_{2} \mathrm{O}\right)$ and as nanoscopic islands $(\mathrm{CuO})$ on the top of that. Time dependent oxide thickness data suggests that oxidation follows the field-assisted growth model at the beginning of the process, as practically immediately an oxide layer of $\sim 2.8 \mathrm{~nm}$ thickness develops on the surface. However, after this initial rapid growth, the local field attenuates and the classical parabolic diffusion limited growth plays the main role in the oxidation. Because of the single crystal facets on the side surface of penta-twinned $\mathrm{Cu}$ nanowires, the oxidation rate in the diffusion limited regime is lower than in polycrystalline films.
\end{abstract}

\section{Introduction}

Oxidation of copper altering its advantageous properties has kept researchers busy for several decades as this metal is being one of the most commonly used one by various industrial sectors. Owing to its outstanding electrical and thermal properties that brought forth numerous applications, this metal gained considerable interest. The unavoidable oxidation of its surface opened new horizons for research and development as formation of p-type oxides with narrow band gap enabled application in solar cells [1], catalysis $[2,3]$ and electronics $[4,5]$. Starting from the beginning of the last century, vast amount of papers on copper and its oxides has been published, revealing the process of native oxide formation on thin films of copper. By the advancement of technology researchers attained control over synthesis procedure allowing to produce copper nanoparticles with well-defined shape and size, thus fostering oxidation studies of such structures under distinct ambient and temperatures.

Though surface characterization of copper thin films remains actual and reports about the facile synthesis of related oxides, e.g. by heating of copper foil, to this day appear in the literature; [6-8] due to intensive development of the field of electronics, optoelectronics, sensors and catalytic materials one-dimensional (1D) materials are in great demand. Therefore, taking into account the size and shape dependent changes in the electrical and optical properties of 1D copper nanostructures, here we disclose the behavioral nature of the native oxidation process on pentagonal copper nanowires. Using a 22-days follow up with transmission electron microscopy, we discuss the oxide growth mechanism considering the generic local electric field-assisted as well as diffusion-limited models and highlight the peculiarities in reference to already reported single and polycrystalline thin films as well as to bulk copper.

\section{Materials and methods}

For the synthesis of copper nanowires, a conventional hydrothermal method was used [9]. Copper chloride dihydrate $\mathrm{CuCl}_{2} \cdot 2 \mathrm{H}_{2} \mathrm{O}$, D-glucose and hexadecylamine (Sigma Aldrich) were used as received without further purification. Copper chloride dihydrate $(0.17 \mathrm{mg})$ was dissolved in deionized water $(80 \mathrm{~mL})$ then $\mathrm{D}$-glucose $(0.39 \mathrm{mg})$ and hexadecylamine $(1.44 \mathrm{mg})$ were added slowly under vigorous stirring. After 5 hours of stirring, a light blue turbid dispersion formed and was placed into a Teflon ${ }^{\circledR}$ lined autoclave (Parr Instrument, Model 4525, $1000 \mathrm{~mL}$ ) and heated to $393 \mathrm{~K}$. After 24 hours of reaction and spontaneous cooling, the obtained brown dispersion was collected, centrifuged at $2500 \mathrm{rpm}$ (990 RCF) for $15 \mathrm{~min}$. The supernatant was discarded and the reddish solid was redispersed in DI water, n-hexane and ethanol to clean the product. The centrifuging, decantation and redispersion cycle was repeated three times in each solvent, and finally, the obtained copper nanowires were kept under ethanol until further analyses to prevent them from oxidation [10].

\footnotetext{
* Corresponding author.

Email address: krisztian.kordas@oulu.fi (K. Kordas)
} 
The formation of oxide layers on copper surface was studied by the means of transmission electron microscopy (TEM). A small aliquot of nanowire dispersion was sonicated in ultrasonic bath for 15 minutes, then drop casted on Ni grids and placed into the TEM column (Jeol FS 2200) after drying. Copper nanowires were analyzed within the time frame of 0-22 days by collecting statistical data about the oxide formation. The samples on Ni grids were kept in a box at $30 \%$ constant humidity after each session of imaging. Images were analyzed using free software EM Measure by Jeol. Since copper surface oxidizes immediately when exposed to ambient oxygen, we were expecting to see some oxide layers on the 0 day of oxidation, formed during the TEM sample preparation period. Each time several nanowires were randomly chosen and assessed by high-resolution imaging and selected area electron diffraction (SAED) in order to assess the evolution of the surface oxides. It is important to note, that because of the curved surface and inhomogeneity of oxide layers, the oxide/metal boundary and the layer thickness are not possible to determine in every location of the samples, thus we collected data only when the lattice fringes of metallic and oxide phases could be clearly identified. For the crystal structure analysis of penta-twinned nanowires a double tilt holder was used. The selected nanowire was tilted along $\mathrm{Y}$ axis about $3^{\circ}$ and the wire was aligned horizontally, in order to prevent precession during following rotation along the $\mathrm{X}$ axis. The zone axis $\langle 115\rangle$ was found by rotation and subsequently recorded SAED images. Due to the penta-twinned structure, the zone axis $\langle 112\rangle$ appears when the NW is rotated with $18^{\circ}$ along the $\mathrm{X}$ axis.

\section{Results and discussion}

Nanowires imaged right after sample preparation ( 0 day of oxidation) showed already a layer of oxide on their surface. While it is commonly known from numerous reports [10-12] that in the early stages of the oxidation process in atmospheric ambient first cuprous oxide forms (because of its lower Gibbs free energy) [13], high-resolution TEM assessment reveals the appearance of both cuprous and cupric ox- ides (Fig. 1, Fig. S1 and Fig. S3). In most of the TEM images, we observe lattice fringes with $\mathrm{D}$-spacing of $\sim 2.5 \AA$, which may be assigned to both the $\mathrm{Cu}_{2} \mathrm{O}(111)$ and $\mathrm{CuO}(11-1)$ planes in the close proximity of the $\mathrm{Cu}$ core. The observed locations of coexisting other fringes with $\mathrm{D}$-spacing values of approximately $3.02 \AA$ and $2.15 \AA$ of $\mathrm{Cu}_{2} \mathrm{O}$ (110) and (200) planes as well as D-spacing value of about $2.75 \AA$ of $\mathrm{CuO}$ (110) indicate that it is the $\mathrm{Cu}_{2} \mathrm{O}$ phase being in direct contact with the metal, and $\mathrm{CuO}$ is mostly on the top of $\mathrm{Cu}_{2} \mathrm{O}$. It is interesting to note, that the long range ordering and the orientation of oxide phases on $\mathrm{Cu}$ as well as on $\mathrm{Cu}_{2} \mathrm{O}$ suggest an epitaxial growth the cuprous oxide on copper and cupric oxide on the top of that.

Islands observed in Fig. 1 are copper oxides (e.g. $\mathrm{Cu}_{2} \mathrm{O}$ in Fig. 1 b, $\mathrm{CuO}$ in Fig. 1d, Fig. 1k and Fig. $\mathrm{S} 1$ and the mixture of both oxide phases in Fig. 1j). Defect sites (grain boundaries, vacancy islands and the edges of pits) are assumed to be the nucleating sites for island formation [14]. We may assume that once a polycrystalline $\mathrm{Cu}_{2} \mathrm{O}$ layer is present on the surface of $\mathrm{Cu}$, some locations favor the nucleation of further oxidation towards $\mathrm{CuO}$ thus giving rise to the formation of islands on the top of the $\mathrm{Cu}_{2} \mathrm{O}$ layer. In addition, further oxidation of $\mathrm{Cu}_{2} \mathrm{O}$ to $\mathrm{CuO}$ may also take place with the help of surface adsorbed water via the formation and subsequent dehydration of metastable $\mathrm{Cu}(\mathrm{OH})_{2}[17-21]$ as we showed earlier in an X-ray photoelectron spectroscopy study [10]. It is worth mentioning that carbon contamination exists on the surface of the nanowires in the forms of a very thin amorphous layer (Fig. 1a, e, h and 1) and small island-like features (Fig. S2).

The overall thickness of the surface oxide does not seem to change significantly with time, in accordance with other works discussing the oxidation of thin films or bulk metal [11-17]. After the rapid formation of the initial oxide layer (within a few tens of minutes) of about $2.8 \mathrm{~nm}$ thickness, further oxide build up is observed to be extremely slow. Within the course of more than 3 weeks of follow up, the measured typical oxide thickness was less than $5 \mathrm{~nm}(3.5 \mathrm{~nm}$ on average). Such a nearly self-terminating growth may be explained by two mechanisms. On the one hand, a local electric field (caused by the contact potential difference between metal and adsorbed oxygen) that helps metal ions

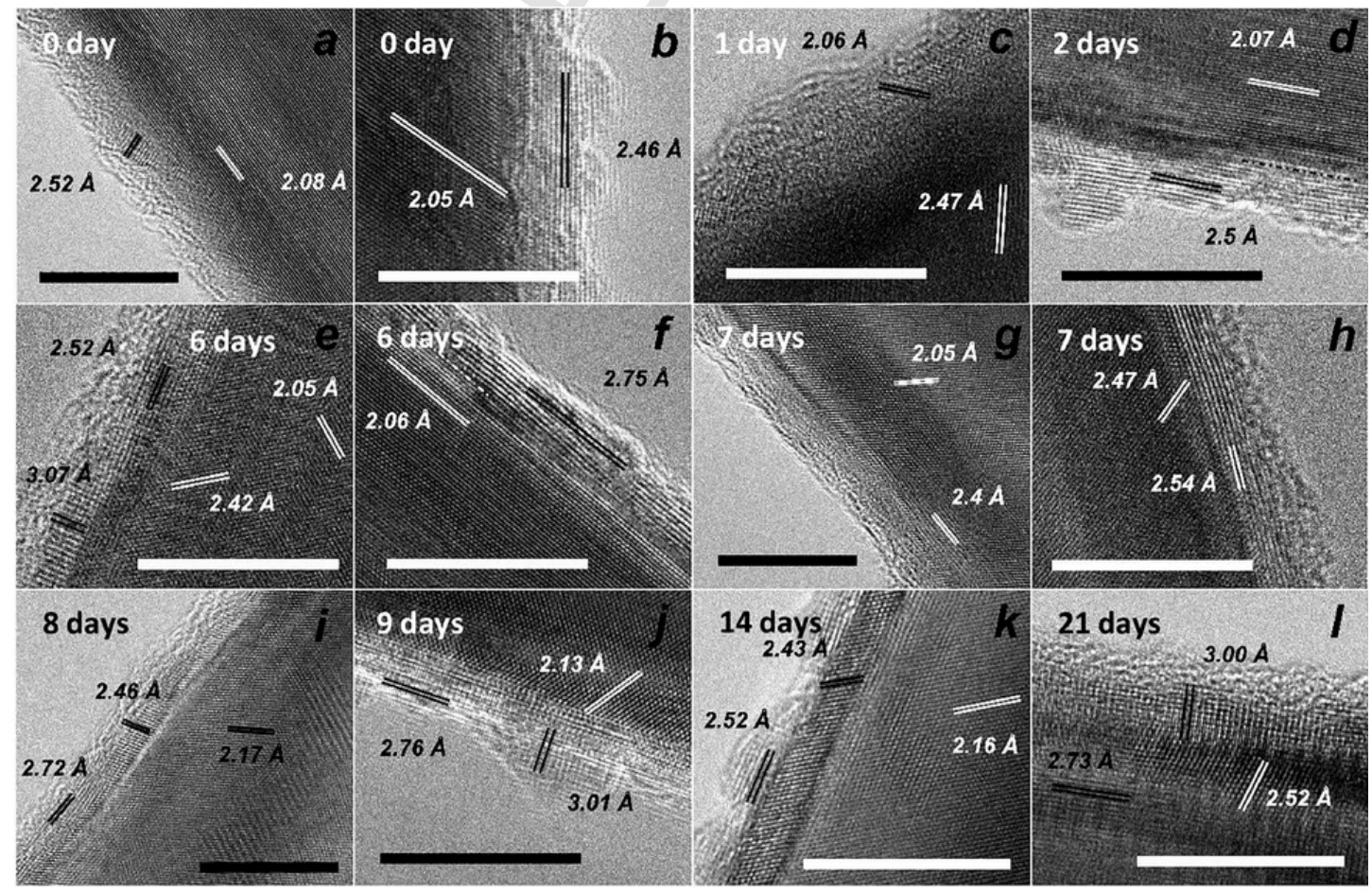

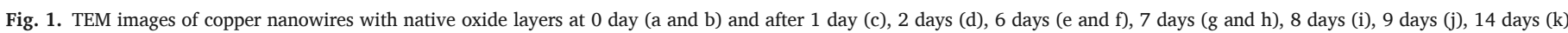
and 22 days (l) of oxidation. Dashed lines (d, f) show the boundary of metal and oxide phases. Scale bars denote $10 \mathrm{~nm}$. 
in drifting from the oxide-metal interface towards the surface is becoming too low to maintain a unidirectional drift of the metal ions (field-assisted model). On the other hand, the rapid formation of a reasonably continuous and compact initial oxide layer, having good oxygen and copper diffusion barrier properties, may slow down and practically inhibit further oxidation of the metallic core of the nanowire (diffusion limited model) [17,18].

To have a better understanding of the oxidation mechanism, we analyze the measured time dependent thickness data by applying both the diffusion limited (Fig. 2a) and field-assisted (Fig. 2b) growth models proposed for $\mathrm{Cu}$ and other common metals.

In our first approach, we fit our plot using a square root function $d(t)=d_{0}+b \cdot t^{0.5}$, in which $d_{0}$ is an offset accounting for the initial oxide thickness and parameter $b=2 D^{0.5}$, where $D$ is the apparent diffusivity of ions in the oxide layer. The value obtained for $D$ is $\sim 3.25 \cdot 10^{-3}$ $\mathrm{nm}^{2} /$ day, which is somewhat lower than those measured by angle-resolved X-ray photoelectron spectroscopy for ion beam deposited [19] and thermally evaporated [20] $\mathrm{Cu}$ films oxidized at room temperature (D' $\sim 18.2 \cdot 10^{-3} \mathrm{~nm}^{2} /$ day and $\mathrm{D}^{\prime \prime} \sim 23.010^{-3} \mathrm{~nm}^{2} /$ day, respectively) (Fig. S3). Our lower diffusivity value is likely because of the direct measurement on a single crystal grain (more precisely on facets of single sub-crystals as we show later), whereas the other data are collected from large surface areas having multiple grain boundaries along which the oxidation has a favored propagation into the specimen surface [14].

On the other hand, as discussed by Cabrera and Mott in their landmark work, [18] metals exposed to oxygen at low temperatures undergo a very rapid (within a period of few minutes or hours) oxidation resulting in a formation of stable oxides having thickness of $2-10 \mathrm{~nm}$. The process is governed by a strong electric field in the oxide film caused by the contact potential difference between metal and adsorbed oxygen, which helps metal ions drifting towards to oxide surface without much influence of the temperature. The process is described by a logarithmic growth law as $1 / d=A-B \cdot \ln (t)$, where the value of slope $B$ is of order $10^{-2}-10^{-1} \mathrm{~nm}^{-1} \cdot{ }^{18}$ Even though the initial oxide layer on our nanowires were around $3 \mathrm{~nm}$, surprisingly when fitting our results with the logarithmic model the fitting parameter $B$ was found to be $\sim 10^{-2}$ $\mathrm{nm}^{-1}$ which value seems to be in good agreement with the oxidation of polycrystalline copper thin films [17].

Looking at the fitting parameters only, our results suggest that the room temperature oxidation of $\mathrm{Cu}$ nanowires may follow both field-assisted and diffusion limited models. However, we have to point out that the initial oxidation, which is governed by the field-assisted growth, is very fast, not taking longer than a few tens of minutes [18]. Therefore, even if the fit gives reasonable parameters for longer oxida-

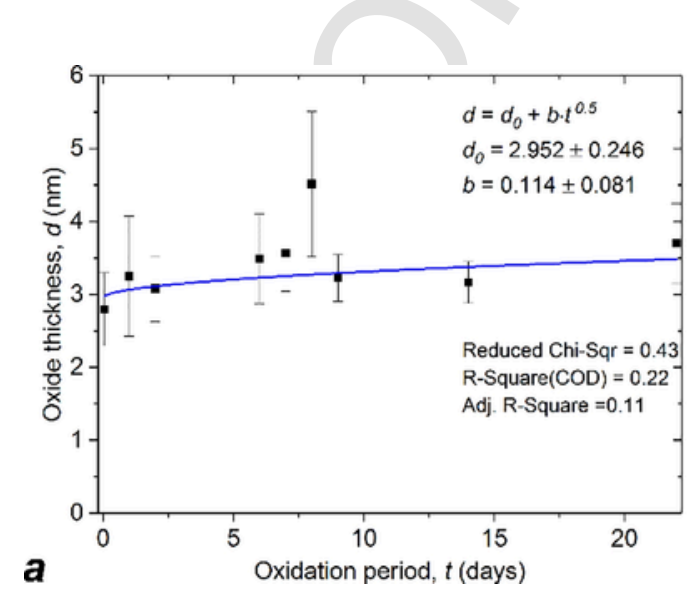

tion periods, it shall not be applied for data collected for days or weeks of periods. The very slow increase of the surface oxide thickness after reaching very quickly the $\sim 2.8 \mathrm{~nm}$ value also dictates the model may not be valid for extended oxidation intervals, and other growth models shall be considered that take into account the formation of vacancies and diffusion of ions in the already formed oxide layer. Accordingly, the validity of the parabolic law fit for our data seems to be more reasonable than the one with the logarithmic model.

Furthermore, it is well known from earlier studies [21,22] that the rate of oxidation highly depends on the crystal structure of the metal and the orientation of exposed surfaces as those determine the formation mechanism of nucleation and growth of oxide islands. Oxidation of (100) facet of single crystal surfaces was reported to be the fastest among the other low index planes such as (110) and (111) [14], which is plausible by considering the high surface energy of the (100) plane [23]. Therefore, to have a better understanding of the oxidation, we carried out selected-area electron diffraction (SAED) on the nanowires to reveal the crystal structure and orientation of their surface. The diffraction patterns resemble superimposed patterns of face-centered cubic lattices and double-diffraction effects caused by twinned sub-crystals $[24,25]$. A copper nanowire oriented in (115) and (112) zone axes (Fig. 3b and 3c, respectively) displays very similar patterns to those reported for penta-twinned nanowires [25] proving that our nanowires are composed of five sub-crystals (numbered as 1, 2, 3, 4, 5 in the inset panels of Figure $3 \mathrm{~d}$ and 3e). These sub-crystals share the same 110 axes and capped by (111) and (100) planes in the apex and on the side surfaces, respectively.

The high area coverage of (100) facets having high surface energy implies that the oxidation of the side surface of the nanowires shall proceed fast. It is actually in line with the observed very rapid initial oxidation reaching $\sim 80 \%$ of the maximum oxide thickness within a few tens of minutes (i.e. in the field-assisted growth regime) in contrast to $\sim 50 \%$ reported for polycrystalline films. On the other hand, we have to note that the measured maximum averaged oxide layer thickness of $\sim 3.5 \mathrm{~nm}$ that developed within 21 days is similar to those reported for oxides grown on polycrystalline thin $\mathrm{Cu}$ films under similar conditions $[12,16,17]$. These results also infer that in the diffusion limited growth regime (after the field-assisted regime) the oxidation of the nanowires becomes slower than those reported for polycrystalline films as it is also reflected by the 6-8 times lower diffusivity values obtained from the corresponding diffusion limited curve fittings (Fig. 2a and Fig. S4) $[12,17]$. This is reasonable by considering the contribution of grain boundaries to a better diffusion of metal ions and oxygen resulting in a faster oxidation of polycrystalline surfaces in contrast to single crystal facets [14].

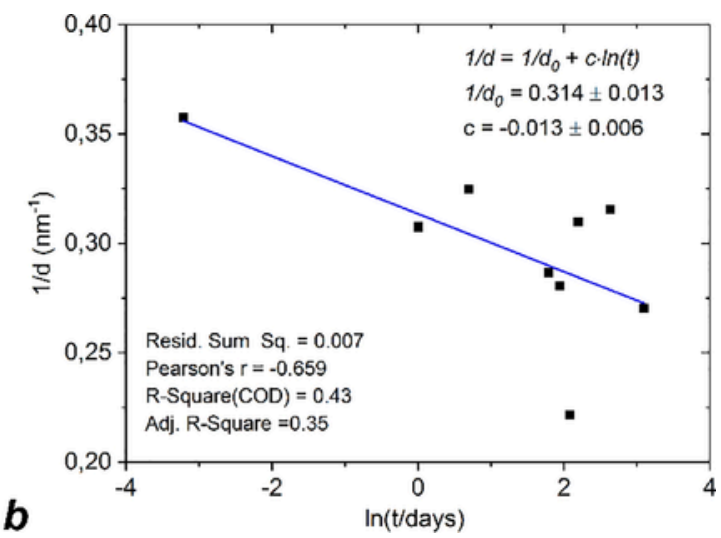

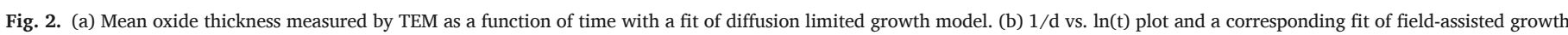
model. 


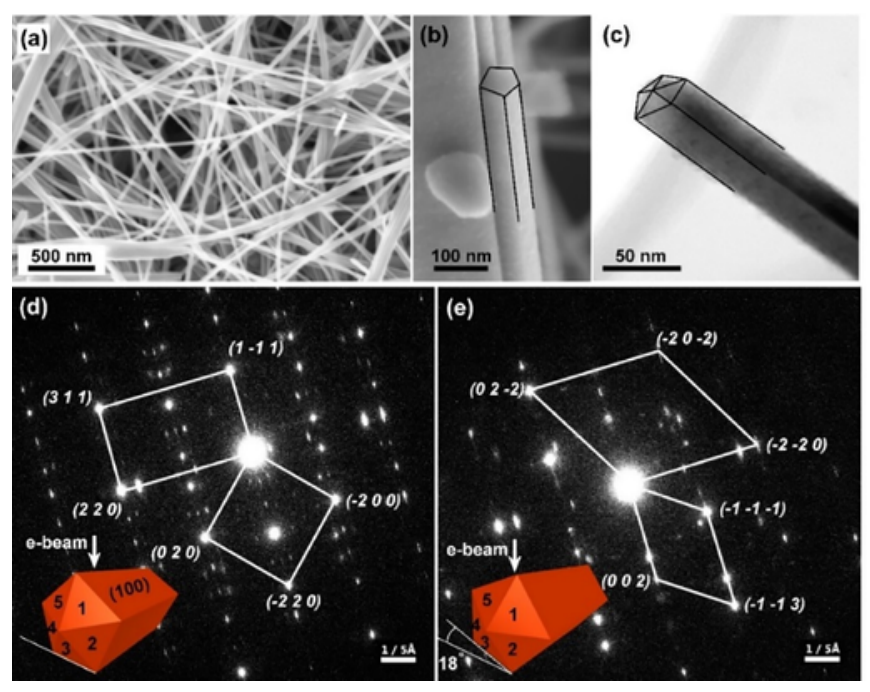

Fig. 3. (a) Low and (b) high magnification scanning electron microscopy images of $\mathrm{Cu}$ NWs. (c) TEM image of a nanowire with five-fold symmetry. SAED patterns of (d) $\langle 115\rangle$ zone axis and (e) zone axis $\langle 112\rangle$, with schematic model of the $\mathrm{Cu} N W$ in the inset.

\section{Conclusions}

In conclusion, our TEM study on the oxidation of hydrothermally grown $\mathrm{Cu}$ nanowires reveals a quick appearance of cuprous oxide followed by cupric oxides on the surface after a few days at room temperature. The crystalline oxides show long range ordering, and their orientation suggests partially epitaxial growth. The oxide thickness variation is very slow after the rapid formation of a thin $(\sim 2.8 \mathrm{~nm})$ oxide layer, and seems to obey the classical parabolic growth model, which sets on after the very rapid field-assisted regime. Although our $\mathrm{Cu}$ nanowires have penta-twinned structure with the high energy (100) facets on the side surface, the oxidation rate in the diffusion limited regime is lower than in polycrystalline films. Our study contributes to the understanding of the oxidation mechanism of $\mathrm{Cu}$ nanowires and serves as a useful background for applications in e.g. catalysis [26-28] and sensors, [28-32] and can help in designing new $\mathrm{Cu}$ based nanomaterials for electronics [33-37].

\section{Other information}

R.H. conceived the experiments and analyzed the results. The manuscript has been discussed and written by R.H, M.M and K.K. Authors declare no competing financial interests.

Supplementary material is available for EFTEM mapping of nanowires, electron diffraction of a nanowire assessed right after the TEM sample preparation; and diffusion limited oxidation rate fitted data retrieved from the literature.

\section{Acknowledgments}

Authors thank Sami Saukko (Nanotechnology and Microscopy Center, University of Oulu) for his assistance in TEM imaging. R. H. ac- knowledges funding received from the University of Oulu Graduate School, Advanced Materials Doctoral Programme. Support received from the Academy of Finland (project SuPlaCat) and University of Oulu (More than Moore research community) is acknowledged.

\section{Supplementary materials}

Supplementary material associated with this article can be found, in the online version, at doi:10.1016/j.susc.2018.03.005.

\section{References}

[1] K. Fujimoto, T. Oku, T. Akiyama, A. Suzuki, J. Phys. Conf. Ser. 433 (2013), 012024.

[2] R. Poreddy, C. Engelbrekt, A. Riisager, Catal. Sci. Technol. 5 (2015) 2467-2477.

[3] J. Li, Z.L. Zhang, Y.J. Ji, Z.Y. Jin, S.Y. Zou, Z.Y. Zhong, F.B. Su, Nano Res. 9 (2016) 1377-1392.

[4] A. Malik, J. Prakash, A. Kumar, A. Dhar, A.M. Biradar, J. Appl. Phys. 112 (2012), 054309.

[5] M. Vaseem, A.R. Hong, R.T. Kim, Y.B. Hahn, J. Mater. Chem. C 1 (2013) 2112-2120.

[6] J.B. Liang, N. Kishi, T. Soga, T. Jimbo, J. Nanomater. 2011 (2011), 268508.

[7] Z.W. Liu, M.L. Zhong, C.M. Tang, IOP. Conf. Ser. Mater. Sci. Eng. 60 (2014), 012022.

[8] F. Beshkar, H. Khojasteh, M. Salavati-Niasari, Materials 10 (2017) 697

[9] M. Mohl, P. Pusztai, A. Kukovecz, Z. Konya, J. Kukkola, K. Kordas, R. Vajtai, P.M. Ajayan, Langmuir 26 (2010) 16496-16502.

[10] R. Hajimammadov, Z. Csendes, J.M. Ojakoski, G.S. Lorite, M. Mohl, K. Kordas, Surf. Sci. 663 (2017) 16-22.

[11] P. Keil, R. Frahm, D. Lutzenkirchen-Hecht, Corros. Sci. 52 (2010) 1305-1316.

[12] J.W. Lim, J. Iijima, Y.F. Zhu, J.H. Yoo, G.S. Choi, K. Mimura, M. Isshiki, Thin Solid Films 516 (2008) 4040-4046.

[13] J.J.D. Leon, D.M. Fryauf, R.D. Cormia and N.P. Kobayashi, Proc. SPIE 9924, Low-Dimensional Materials and Devices 992400 (2016).

[14] C. Gattinoni, A. Michaelides, Surf. Sci. Rep. 70 (2015) 424-447.

[15] M. Ramirez, L. Henneken, S. Virtanen, Appl. Surf. Sci. 257 (2011) 6481-6488.

[16] J. Iijima, J.W. Lim, S.H. Hong, S. Suzuki, K. Mimura, A. Isshiki, Appl. Surf. Sci. 253 (2006) 2825-2829.

[17] I. Platzman, R. Brener, H. Haick, R. Tannenbaum, J. Phys. Chem. C 112 (2008) 1101-1108.

[18] N. Cabrera, N.F. Mott, Rep. Prog. Phys. 12 (1949) 163-184

[19] Note: $\mathrm{D}^{\prime}$ is calculated by digitizing and fitting data reported in Lim J.-W. et al. Thin Solid Films 516, 4040-4046 (2008).

[20] Note: $\mathrm{D}^{\prime \prime}$ is calculated by digitizing and fitting data reported in I. Platzman et al. J. Phys. Chem. C, 112, 1101-1108 (2008).

[21] Y.P. Timalsina, M. Washington, G.-C. Wang, T.-M. Lu, Appl. Surf. Sci. 363 (2016) 209-216.

[22] G. Zhou, J.C. Yang, J. Mater. Res. 20 (2005) 1684-1694.

[23] M.I. Baskes, Phys. Rev. B 46 (1992) 2727-2742.

[24] I. Lisiecki, A. Filankembo, H. Sack-Kongehl, K. Weiss, M.P. Pileni, J. Urban, Phys. Rev. B 61 (2000) 4968-4974.

[25] H.-J. Yang, S.-Y. He, H.-Y. Tuan, Langmuir 30 (2014) 602-610.

[26] G. Fan, L. Yu, L. Dong, ECS Trans. 53 (2013) 29-33.

[27] Y. Zheng, J. Liang, Y. Chen, Z. Liu, RCS Adv. 4 (2014) 41683-41689.

[28] J.S. Easow, T. Selvaraju, Electrochim. Acta 112 (2013) 648-654.

[29] H. Shokry Hassan, A.B. Kashyout, I. Morsi, A.A.A. Nasser, H. Abuklill, Sens. Bio-Sens. Res. 5 (2015) 50-54.

[30] L. Ju, G. Wu, B. Lu, X. Li, H. Wu, A. Liu, Electroanal. 28 (2016) 2543-2551.

[31] X. Xu, R. Wang, P. Cheng, X. Lu, L. Shi, J. Sun, ACS Appl. Mater. Interf. 9 (2017) 14273-14280.

[32] A.M. Stortini, L.M. Moretto, A. Mardegan, M. Ongaro, P. Ugo, Sens. Actuat. B 207 (2015) 186-192.

[33] A.R. Rathmell, M. Nguyen, M. Chi, B.J. Wiley, Nano Lett. 12 (2012) 3193-3199.

[34] X. Luo, G.A. Gelves, U. Sundararaj, J.L. Luo, Can. J. Chem. Eng. 93 (2013) 630-637.

[35] Z. Niu, et al., J. Am. Chem. Soc. 139 (2017) 7348-7354.

[36] L. Shi, R. Wang, H. Zhai, Y. Liu, L. Gao, J. Sun, Phys. Chem. Chem. Phys 17 (2015) 4231-4236.

[37] J. Song, J. Li, J. Xu, H. Zeng, Nano Lett. 14 (2014) 6298-6305. 